\title{
PNEUMATIC POSITIONING SYSTEM FOR PRECISION ASSEMBLY
}

\author{
Martin Freundt, Christian Brecher, Christian Wenzel, Nicolas Pyschny \\ Fraunhofer IPT, Steinbachstraße 17, 52074 Aachen, Germany \\ martin.freundt@ipt.fraunhofer.de
}

\begin{abstract}
Micro assembly is typically characterised by positioning tolerances below a few micrometers. In the case of a hybrid micro system assembly, such as optical glass fibres, micro ball lenses or micro probes for measurement tasks, even positioning accuracies in the sub-micrometer range have to be achieved. Due to the need for highly accurate assembly systems and extensive alignment procedures the assembly of hybrid microsystems is characterised by customised solutions. In this context the Fraunhofer IPT develops a concept on how to realise a highly flexible, fast and cost-efficient hybrid assembly system, consisting of a conventional assembly device and an active assembly head.

The active assembly head will be pre-positioned by imprecise but dynamic conventional handling devices like an industrial robot. By means of its integrated 6axes fine positioning system and its sensor system it will be able to first detect position and orientation deviations and second compensate the deviation and execute the final positioning and alignment of the micro part. In this context, a matchbox-sized air bearing stage with an integrated non contact interface for a transfer of pneumatic energy between stage and slides was conceived, allowing ultra precise and frictionless guidance for travel ranges up to $3 \mathrm{~mm}$. In order to apply this distinct design to the whole system, even actuators and sensors must be wireless or pneumatically driven in order to ensure a friction free stage movement.

In this context the requirements caused by a conventional pre-positioning device will be discussed. It will be shown that travel ranges of about $1 \mathrm{~mm}$ must be achieved in order to allow a reliable and safe use of the hybrid handling device. Based on that analysis, a design of a friction free, damped pneumatic actuator design as well as a concept of a pneumatic sensor will be presented. Both components will target a travel range of $1 \mathrm{~mm}$ combined with a resolution in the sub-micron range.
\end{abstract}

\section{Introduction - Initial Context}

Technological miniaturisation is a major trend and thus a key characteristic of future product innovations [1]. Highly sophisticated machining processes such as 
ultra precision diamond cutting, micro-erosive forming and lithographic processes enable the manufacturing of microscopic components as small as $100 \mu \mathrm{m}^{3}$. These highly precise components are in most cases mounted onto larger macroscopic components, to be incorporated into innovative medical, telecommunication or sensor technology products.

Key factors of micro systems production are technologies for the automated handling, alignment and assembly of micro parts. Although available systems for these purposes are sufficiently precise, they are, compared to macroscopic assembly equipment, highly specialised and inflexible, i.e. can hardly be applied or adapted to different assembly tasks. For the flexible automation of macro assembly processes a wide range of standardised handling systems like industrial robots is currently available. But as yet, even the most precise industrial robots cannot be applied to micro assembly processes with accuracy requirements in positioning and adjustment of less than one micrometer.

The conditions that apply to micro component assembly are generally very different to those of macro assembly, e.g. thermal fluctuations, vibrations and the fundamental tolerances of the tools and equipment have a strong influence on assembly results and product quality.

The lack of versatile, modular standard components for micro component handling increases the complexity of an automated micro assembly in terms of engineering, and mechanical design efforts as well as the controls needed to customize independent and oftentimes incompatible automation solutions. As a result, the field of micro assembly keeps being dominated by manual assembly operations, especially for the production of small and medium lot sizes, where highly specialised automation components cannot be operated cost-effectively.

It therefore becomes very difficult to integrate micro assembly operations into production processes related to macroscopic products, and it remains indispensible to synchronise separated microscopic and macroscopic assembly processes, which is both costly and technically complex.

\section{State of Technology and Technical Concept}

For micro assembly purposes many different positioning and alignment systems have been developed. In order to be able to deal with the entire handling process, an assembly system must incorporate the following process steps in one device:

- Picking up the micro part from a component holder,

- Moving the micro part to the assembly location,

- Positioning and aligning the micro part.

Highly precise positioning systems for the assembly of micro systems, individual components such as positioning axes as well as highly precise actuator and sensor components used to build specialised equipment are well established and readily available. Examples include the systems from Klocke Nanotechnik, MicRohCell (Rohwedder AG), AutoPlace 400 (Sysmelec), RP series (Mitsubishi), and micropositioning stages (Physik Instrumente, Mechonics). 
Further systems for micro assembly can be found in the areas of surface mount devices (SMD) and die bonders. SMD assembly is characterised by very high dynamics, but only achieves a precision of $40-50 \mu \mathrm{m}$, and offers no more than 3 degrees of freedom (DOF). Die bonders for industrial chip assemblies are Cartesian pick-\&-place systems with integrated sensors (mostly cameras) to achieve exact positioning of microelectronic components. However, although die bonders offer accuracies down to $\pm 1 \mu \mathrm{m}$ these systems are very capital intensive and restricted in their available degrees of freedom due to the Cartesian setup [1].

The typical serial Cartesian design of gantry assembly systems, based on individual, high precision but relatively massive axis components, inevitably leads to large solid cantilever structures. Such very massive systems usually include granite elements with a closed-off structure. This design reduces the usability of the systems to a few specific applications. Depending on the number of assembly partners involved, the type of packaging and storing as well as the size of micro parts and component holders, motion paths for all three process steps can easily exceed $400 \mathrm{~mm}$.

Generally, conventional assembly systems have enough structural rigidity to work in spaces of some $100 \mathrm{~mm}^{3}$ with a positioning accuracy in the sub-micrometer range, but have a very small working space compared to their large base and frame size. Large structures are associated with problems in terms of thermal stability. Systems that are sensitive to thermal influences are hardly suitable for applications where the thermal conditions are largely undefined as is the case in macroscopic production processes. With mostly less than six degrees of freedom and a common system structure that limits the construction space, and thus prevents a flexible integration into the flow of material, the assembly process must be redesigned and modified accordingly to fit the assembly device.

Indeed, more flexible, macroscopic robot systems with six DOF only achieve a repeating accuracy down to $20 \mu \mathrm{m}$. They are therefore unsuitable for the assembly of micro components which are about 100 to $200 \mu \mathrm{m}$ in size. For such products such as sensor tips for micro probes or surgical suture material for complex surgical procedures (e.g. eye operations) - a positioning accuracy in the sub-micrometer range is necessary. These robots do not fulfil the requirements of alignment procedures for larger components, such as optical components of a diode laser.

Available stationary positioning stages providing the required accuracy for the alignment in more than three or four DOF restrict the flexibility of the assembly system (as positioning and alignment processes have to be located at the station), and cause difficulties in integrating the separated module controls into a common system control. Therefore, different approaches of mounting portable alignment modules to conventional robot systems have been pursued to achieve maximum flexibility regarding assembly and positioning tasks as well as different products and components [2].

In this hybrid concept an active alignment module is mounted to the imprecise but dynamic handling robot serving as a portable multi-axis alignment device. The head will be pre-positioned within the large work space of the robot, reference its position and orientation at the assembly location and compensate position and alignment errors with micrometer-accuracy. Thereby the necessary precision for the alignment of optical components can be realised at the tip of the assembly head 
compensating for positioning errors and guaranteeing full system functionality (Figure 1).

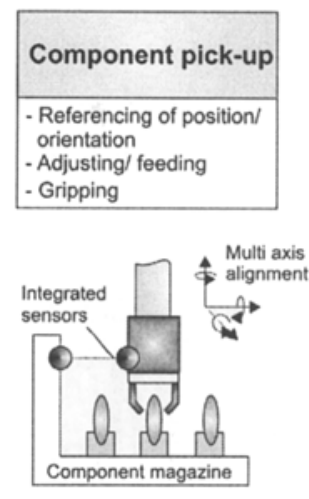

Micro workspace
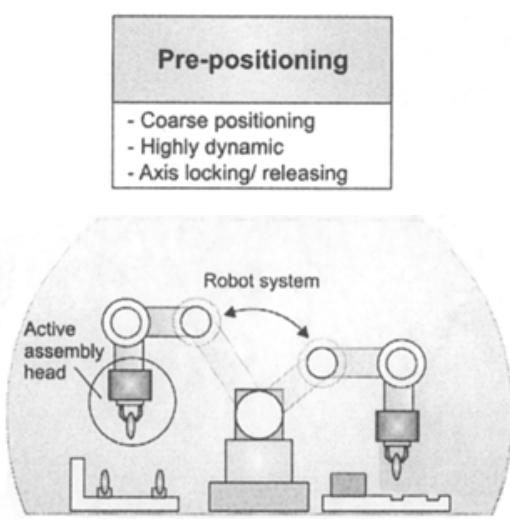

Macro workspace
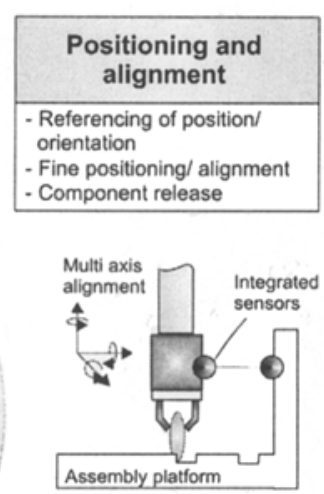

Micro workspace

Fig. 1. Hybrid assembly system concept with active assembly head

This hybrid system concept combines the advantages of standardised, conventional robot systems for macroscopic handling processes, i.e. the large work space, high availability and dynamics with the required accuracy for micro assembly tasks. By a flexible changeover between macroscopic and microscopic operating environments a fast integration of high-accuracy assembly and alignment processes into conventional assembly lines will be enabled. This approach has already been adopted in research projects to design fine adjustment units for a very limited range of specialized assembly applications. As examples for these specialised units investigations were conducted into the active adjustment of single mode glass fibres and the camera-aided positioning of electronic components. [3, 4]

To gain broader acceptance of the hybrid concept, a universally applicable active assembly head has to be developed that is qualified for mobile mounting on standard pre-positioning units (e.g. industrial robots) allowing the flexible application of different end-effectors' geometries for multiple highly-precise assembly tasks. Resulting standardised micro assembly systems that are able to cope with a wide range of micro assembly operations would help to shorten cycle times, increase the flexibility of the positioning and adjustment systems and improve the set-up process. These improvements could significantly increase the efficiency of automated micro assembly processes, opening up a new range of micro system applications. 


\section{Deduction of Design Requirements}

In order to improve the precision of an industrial robot system by means of an active assembly head mounted in series, system vibrations caused by active robot drives have to be suppressed or compensated. Measurements show that in conventional systems the stroke of these drive vibrations can range to several micrometers (Figure 2). Vibrations can significantly be reduced when the robot is operating with activated brakes and deactivated drives. The brake activation prevents any agitation of the robot's structure, but results in a load alternation from the drive train to the brakes, associated with non-reproducible movements in the robot's joints causing the robot arm to sag.

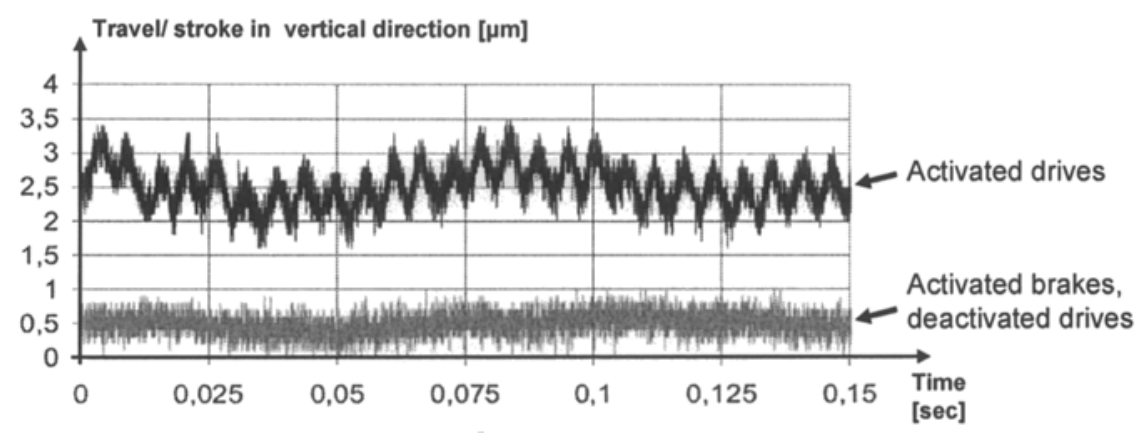

Fig. 2. Vibrations in a 6-axis-robot (arm acting against gravity)

Practical investigation carried out on a 6-axis-robot with an arm length of approx. $600 \mathrm{~mm}$ have shown that the robot hand loses approx. $300 \mu \mathrm{m}$ in height and moves $0.04^{\circ}$ from position when brakes are activated. Variations range $\pm 100 \mu \mathrm{m}$ and $\pm 0.02^{\circ}$ due to changing frictional and bracing characteristics of the robot arm.

Thus, a hybrid system of a conventional robot and an active assembly head can only perform stable and reliable positioning operations if the fine positioning axles are designed to cope with travel ranges of at least $1000 \mu \mathrm{m}$. In this case, the assembly head can compensate for sagging of between 200 and $500 \mu \mathrm{m}$, still providing enough travel (several $100 \mu \mathrm{m}$ ) to carry out positioning and alignment operations.

\section{Design Approach}

As part of the SFB 440 project "Assembly of hybrid micro systems", current research activities at Fraunhofer IPT aim at the development of an assembly head that meets the aforementioned criteria, and includes an integrated, highly precise, compact and robust fine positioning unit with 6 degrees of freedom and integrated sensors for referencing at the assembly location. The work covers the development 
of system components in line with the identified requirements, the design of the system structure as well as the development of the controls needed to regulate the interaction between the assembly head and the conventional pre-positioning system. Main tasks in this context are the appropriate miniaturization of guideways as well as the design of compact actuator and sensor components.

State-of-the-art design of portable fine positioning systems incorporates solid hinges that so far only achieve a maximum travel range of about $200 \mu \mathrm{m}$ preventing the formation of flexible positioning systems for various micro assembly applications. The hybrid concept requires a mechanical design that enables travel ranges about $1 \mathrm{~mm}$ with angles up to $1^{\circ}$, and possesses an integrated sensor system to reference and control the assembly head.

This in a first step necessitates the development of a guideway technology that can perform compensational and positioning movements with sub-micrometer accuracy over the required travel range. As solid hinges do not fulfil the deducted requirements the design approach focuses on the miniaturisation of air bearing technology and its qualification for the set-up of a 6 -axis positioning module.

\section{Guideway with Air Bearings and Pneumatic Energy Transmission}

Fundamental research activities pursue the development of a friction-free, miniaturised guiding unit with air bearings and up to $3 \mathrm{~mm}$ travel range.

The downsizing of an axis with air bearings for the application in highly precise motion guides requires several design changes towards miniaturisation. The miniaturisation of vacuum pre-loaded air bearings to a pad diameter of $14 \mathrm{~mm}$ for example demands a reduction of the bearing gap from already very narrow 2 - $4 \mu \mathrm{m}$ to under one micrometer in order to achieve similar rigidity and damping properties.

Due to the technically highly challenging reduction in bearing clearance, it becomes clear that - due to restrictions in manufacturing accuracy - it will be difficult and expensive to miniaturize the air bearings without compromising on bearing rigidity and damping.

A prototype in operation at the Fraunhofer IPT has a relatively large bearing gap of approx. $2 \mu \mathrm{m}$. With a bearing pad diameter of $14 \mathrm{~mm}$ this leads to an operating point with relatively low rigidity and damping. Nevertheless technical feasibility of the miniaturised air bearing technology with vacuum pre-loading meeting the requirements in terms of accuracy and travel range of at least $1 \mathrm{~mm}$ could be demonstrated (Figure 3). Despite the large bearing clearance, the bearing stability could be proved: according to the calculations from W.J. Barz [5], the operating point lies below the region of bearing instability for average load ratings, while rigidity and damping are however significantly lower than for conventional, nonminiaturised bearing stages where the load capacity has been optimised for greater stability. 
In order to qualify the air bearings for a highly precise multi-axial positioning system, bearing loads have to be reduced to an extent where the negative effects of low rigidity and damping on the system behavior can be tolerated.

In the context of the presented design approach, bearing loads arise from three factors: the acceleration of the carrying robot systems, the interfering forces created by the actuators and sensors (parasitic shearing forces and moments), and the interfering forces caused by the rigid component wiring.

This demands all components - the guides, the actuators, the sensors and the system supply lines - to be designed with regard to light weight, i.e. low inertia, and resistance-free signal, energy and fluid transmission. If the entire system can be build light enough that varying loads do not affect operability of the fine positioning system, it becomes possible to implement the miniaturised air bearing technology without reducing critical design parameters, like bearing clearance.
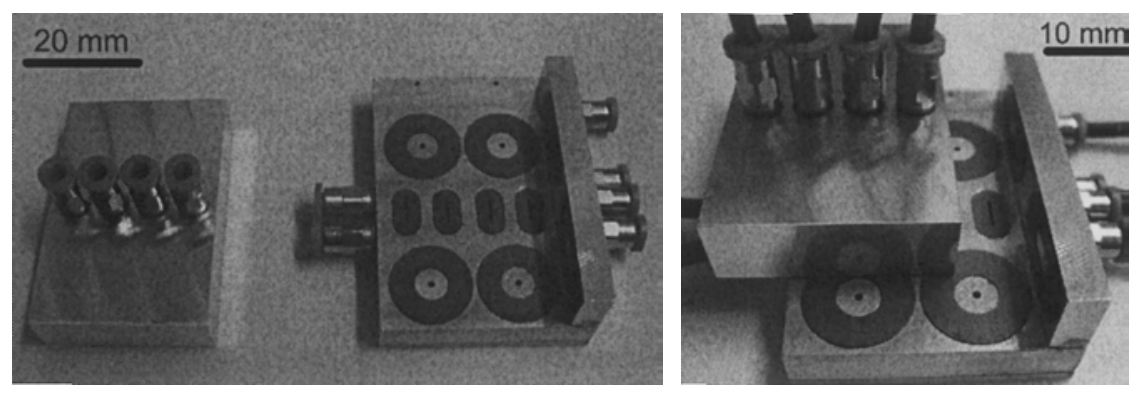

Fig. 3. Bearing pads in a matchbox-sized prototype air bearing with vacuum pre-loading

In this context, the Fraunhofer IPT developed a concept for non-contact compressed air transmission between guideway and slide. The transmission of compressed air via special ducts in the bearing surface avoids friction, force and hysteresis. The transmission ducts are found in both the slide (entrance) and the guideway (exit) aligned opposite to one other. The outlet in the guideway - an elongated slot - allows for axle movement without disconnecting the compressed air or vacuum supply lines between guide and slide. The transition between entrance and exit outlet is sealed and throttled by a non-contact annular gap. The low sealing gap, corresponding to the bearing gap, reduces leaks to a minimum and ensures contact-free operation, without friction, force or hysteresis.

The prototype shown in Figure 3 demonstrates that five serially stacked guideways with air bearings can be supplied with, and run on compressed air without any rigid supply lines between stage and slide. Hence, each guideway can be operated without affecting the others, thereby reducing the requirements on actuator force and rigidity, which in turn enables the miniaturisation of other components. 


\section{Design of a Pneumatic Actuator}

Primarily objective of the pneumatic actuator design is highest possible accuracy. In accordance to the aforementioned requirements, the actuator system has to be small and lightweight, as well as frictionless to prevent negative side effects, like stickslip.

Conventional designs prove unusable due to contacting sealing elements. Possible contactless and hence frictionless solutions are bellow- and membrane structures, both working without contacting parts. Membrane structures have the advantage of exhibiting high stiffness in one specific direction. But the resulting excellent guideway abilities unfortunately incorporate very small travel distances in the range of a few hundred micrometers. Bellows on the other hand do not have guiding abilities, but even in very small dimensions they allow travel distances in the range of millimetres, suitable to meet the requirements discussed before.

For both designs - bellow or membrane - once the actuator works friction free, no damping occurs, and the mechanical structure resembles a spring. Additionally the compressed air in a pneumatic actuator has a similar behaviour with very low damping and almost no mass.

A completely friction free pneumatic actuator system is not suited for highly precise positioning, as with very low damping existing disturbing forces would cause vibrations and oscillation of the device. Therefore, a damping element must be integrated into the pneumatic actuator which works without causing stick-slip effects, but still damping the oscillations caused by internal and external forces.

An approach, conducted at the Fraunhofer IPT is the integration of an oil based damping device. Because of the required accuracy in the air bearing stages, an oil based device has to be concealed in order to avoid contamination of ultra-precisely manufactured components. Therefore, in contrast to a squeeze film damper arranged in direction of the stage movement, the developed solution is based on an oil filled bellow - the same technology used for the pneumatic actuator. The developed sealable, contamination free damping technology is expected to have significant advantages in long term stability of the system behaviour.

To avoid any parasitic forces the damping and actuating forces of the pneumatic actuator have to have exactly the same contact point and direction. This can be realized by a setup, where the actuator bellow is integrated inside the damping bellow (Figure 4).

This design allows optimised exploitation of construction space and the reduction of parasitic forces. In order to maintain a sealed system, a third bellow is needed as fluid reservoir, whereby the pipe between damping bellow and reservoir bellow works as a resistor causing the damping effect. Applying the bellow technology to a pneumatic actuator and a fluidic damper, the design of a stick-slip free, but damped actuator system is made possible.

Every pneumatic actuator, based on a piston, membrane or bellow design is in fact a pressure-travel converter. Thus, once high precision in travel is required, high precision in the adjustment of the pressure is essential. The specific precision depends on the targeted travel range and the stiffness of the bellow system described before. 


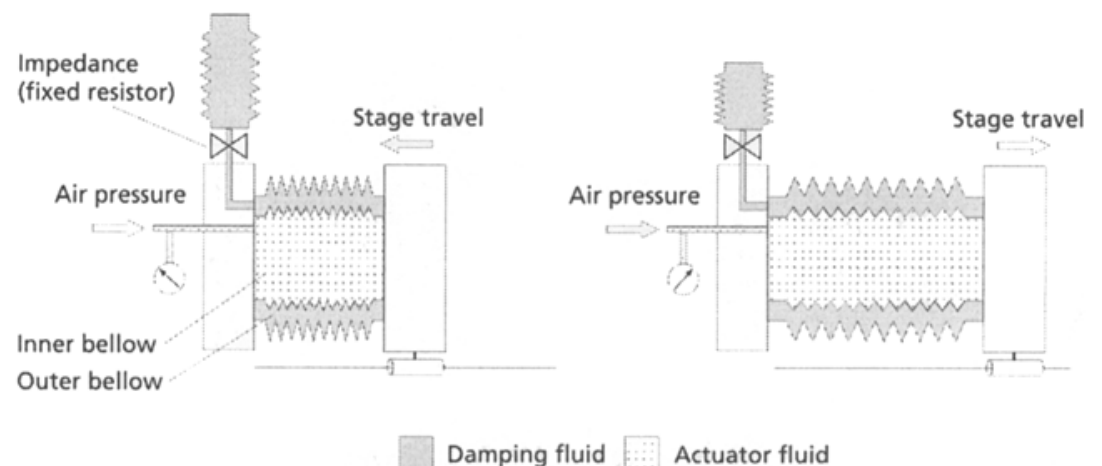

Fig. 4. Stick-slip free, oil-damped pneumatic driven actuator

For estimating calculations of the bellow system's stiffness, both bellows and compressed air can be represented by a conventional spring component. Therefore following simplification will be presumed: Each bellow is represented by the stiffness coefficients $c_{A}, c_{D}, c_{R}$. The inner volume including a part of the pipe system is represented by the stiffness coefficient $c_{\text {air }}$. The over all stiffness can be calculated with following equation:

$$
\frac{1}{c_{g e s}}=\frac{1}{c_{a i r}+c_{A}+c_{D}+c_{R}}
$$

The achievable travel range $\mathrm{X}$ can be calculated as:

$$
X=\frac{F}{c_{\text {ges }}}=\frac{\left(P_{\text {Actuatorbellow }}-P_{0}\right) \cdot\left(A_{\text {Actuatorbellow }}-A_{\text {Damperbellow }}\right)}{c_{\text {ges }}}
$$

For a required travel range of about $1 \mathrm{~mm}$ and a step width of $0,1 \mu \mathrm{m}$ following sample calculation - based on the assumption, that the common air supply delivers a pressure of 6 bar absolute - shows the requirements on a pressure adjustment device.

$$
\frac{s}{\Delta s}=\frac{p_{\text {aktuator }}-p_{0}}{\Delta p} \Rightarrow \frac{1 \mathrm{~mm}}{0,0001 \mathrm{~mm}}=\frac{6 \mathrm{bar}-1 \mathrm{bar}}{\Delta p} \Leftrightarrow \Delta p=0,0005 \mathrm{bar}
$$

This example shows that running the actuator based its pressure-travel converter principle requires a pressure resolution of 0,5 mbar. As the systems underlies operating conditions with pressure variations in air supply and environment, which will have significant impact on step width and achievable accuracy of the actuator, a closed-loop positioning control is necessary.

This demands respective sensor solutions for position recognition supporting the overall lightweight design approach. 


\section{Pneumatic Sensor Concept}

In correspondence with the approach of reduced system size, mass and actuator forces, further investigations are conducted into the research of force-free and frictionless pneumatic based sensor systems which can be interpreted by measuring air flow or pressure to unify fluidic and energetic supplies for sensor, air bearing and actuator components.

The common measuring principle nozzle and deflector as it is used in pneumatic valves (Figure 5 left) can not be used in this case. Preliminary tests have shown the nozzle-deflector principle works best in a region of a few micrometers. For the use as absolute measuring device, precision and range are interdependent, mostly determined by the available digitalisation resolution, interferences in the signal, and the limitations of the physical principle. The requirement for travel ranges over 100 $\mu \mathrm{m}$ does hence not allow the use of this technology. Therefore, a relative measuring pneumatic sensor principle is needed. Inspired by the common principle of a linear measuring scale used in wide range of applications, the following concept was developed (Figure 5 right).
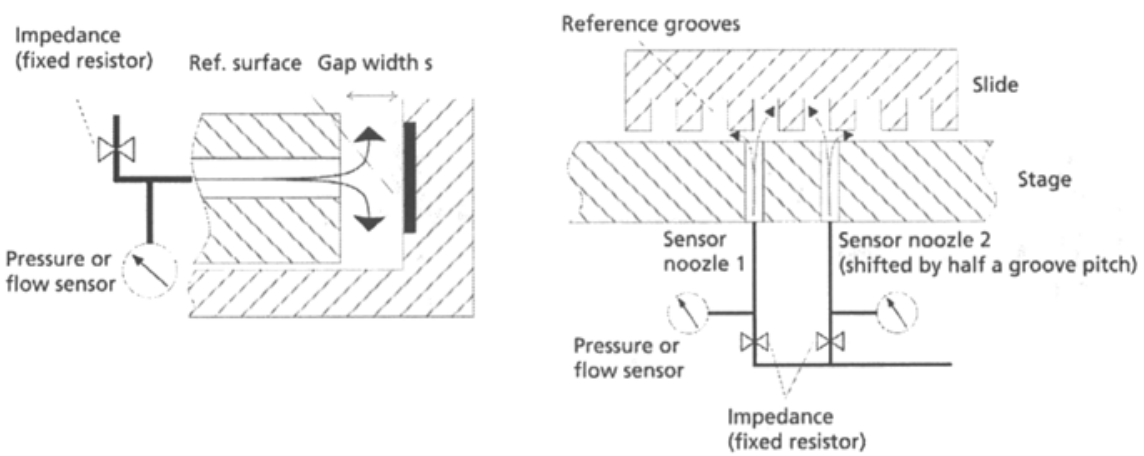

Fig. 5. Pneumatic sensor concept

Therefore it is used to scan a surface of the slide in which small grooves are inserted. Once the slide moves, the impendence in front of the nozzle is influenced by the groove structure. With a groove in front of the nozzle the resistance is reduced and a high air flow is established. If the nozzle is almost closed by the bar, the flow is reduced. The system thereby produces a pressure or air flow signal, similar to that which is generated in conventional optical linear measurement scale. Using two nozzles, placed with an offset compared to the groove structure, it is possible to read the direction of the movement. 


\section{Outlook}

The technology of pneumatic based actuator and sensor systems presented here is currently being investigated in terms of its ability to be integrated into a miniaturised stage system. Therefore the Fraunhofer IPT is already investigating to what precision such system can be utilised. The next stage in this investigation is to set up a test stand in order to prove the technological feasibility and characterise the achievable precision. The main research issue will be how to handle the capacity effects caused by the compressibility of compressed air, which influence both actuator and sensor components.

\section{Acknowledgements}

The authors would like to thank the »Deutschen Forschungsgemeinschaft (DFG)« for supporting the »Greifer und Montagemaschinen« research project as part of the special research topic SFB 440 »Assembly of hybrid micro systems«.

\section{References}

[1] M. Höhn: Sensorgeführte Montage hybrider Mikrosysteme, Dissertation, München, Technische Universität, 2001

[2] A. Schubert, H.-J. Koriath: Precision-Tilt-Gripper with rigid 3d-structure. In: Proceedings of the $4^{\text {th }}$ euspen international conference, Glasgow, 2004

[3] C. Peschke: Mehr-Achs-Mikrogreifer zur Handhabung von Drähten und Fasern, Dissertation, RWTH Aachen 2007

[4] J. Hesselbach: mikroPRO - Untersuchung zum internationalen Stand der Mikroproduktionstechnik. Essen: Vulkan-Verlag, 2002

[5] W.J. Bartz: Luftlagerungen - Grundlagen und Anwendungen. Esslingen: expertverlag, 1993, ISBN 3-8169-0992-2 